EPJ manuscript No.

(will be inserted by the editor)

\title{
Nucleon electroweak form factors in a meson-cloud model
}

\author{
B. Pasquini and S. Boffi \\ Dipartimento di Fisica Nucleare e Teorica, Università degli Studi di Pavia and INFN, Sezione di Pavia, Pavia, Italy \\ Received: date / Revised version: date
}

\begin{abstract}
The meson-cloud model of the nucleon consisting of a system of three valence quarks surrounded by a meson cloud is applied to study the electroweak structure of the proton and neutron. The electroweak nucleon form factors are calculated within a light-front approach, by obtaining an overall good description of the experimental data. Charge densities as a function of the transverse distance with respect to the direction of the three-momentum transfer are also discussed.
\end{abstract}

PACS. 12.39.-x Phenomenological quark models - 13.40.Gp Electromagnetic form factors

\section{Introduction}

The relevant role played by the meson cloud of the nucleon is clearly manifest in the study of the low-energy electromagnetic structure of the nucleon. Here we want to consider the role of the meson-cloud in the calculation of the electroweak form factors of the nucleon within a relativistic light-front approach where the nucleon light-cone wave function (LCWF) is derived by performing a baryonmeson Fock-state expansion. In the one-meson approximation the nucleon state $|\tilde{N}\rangle$ is pictured as being part of the time a bare nucleon, $|N\rangle$, and part of the time a baryonmeson system, $|B M\rangle$. The bare nucleon is formed by three valence quarks identified as constituent quarks, while the baryon-meson system is assumed to include configurations with the baryon being a nucleon or a Delta and the accompanying meson being a pion as well as a vector meson such as the $\rho$ or the $\omega$. The calculation of the electroweak form factors of the proton and neutron is illustrated in Sect. 2. and the results are presented and discussed in Sect. 3 .

\section{Electroweak form factors of the nucleon in the meson-cloud model}

The problem of considering the meson cloud surrounding a system of three valence quarks has been addressed already in the past in a variety of papers $[1,2,3,4,5$, and it has recently been revisited to study the generalized parton distributions [6] and the electroweak form factors of the nucleon [7. We refer to these last two works for a detailed explanation of the derivation of the LCWF of the nucleon in the meson-cloud model in terms of a bare (three quark) and a baryon-meson (five quark) component, while in the following we review the main steps of the convolution formalism for the calculation of the electromagnetic and axial form factors of the nucleon.
The Dirac $\left(F_{1}\right)$ and Pauli $\left(F_{2}\right)$ form factors of the nucleon are given by the spin conserving and the spin-flip matrix elements of the vector current $J_{V}^{+}=J_{V}^{0}+J_{V}^{3}$

$$
\begin{aligned}
F_{1}\left(Q^{2}\right) & =\left\langle\tilde{p}+\tilde{q}, \frac{1}{2}\left|J_{V}^{+}\right| \tilde{p}, \frac{1}{2}\right\rangle, \\
\left(q_{x}+i q_{y}\right) F_{2}\left(Q^{2}\right) & =2 M_{N}\left\langle\tilde{p}+\tilde{q},-\frac{1}{2}\left|J_{V}^{+}\right| \tilde{p}, \frac{1}{2}\right\rangle,
\end{aligned}
$$

where $q$ is the momentum transferred, and $Q^{2}=-q^{2}$. The corresponding relation between the axial current $J_{A}$ and the axial form factor $G_{A}\left(Q^{2}\right)$ is

$$
G_{A}\left(Q^{2}\right)=\left\langle\tilde{p}+\tilde{q}, \frac{1}{2}\left|J_{A}^{+}\right| \tilde{p}, \frac{1}{2}\right\rangle .
$$

The convolution formalism derived in the following formulas apply for the electomagnetic as well as for the axial current, and we will use $J^{+}$for either one of the two currents. The calculation of the form factors is conveniently done in a coordinate frame with $q^{+}=0$, where the current matrix elements can be computed as a simple overlap of Fock-space wave functions with the same number of partons, with all off-diagonal terms involving pair production or annihilation by the current or vacuum vanishing. In the present meson-cloud model, we need to consider the contributions from the diagonal overlap between the bare-nucleon state, on one side, and the $B M$ components, on the other side. Furthermore, the current is a sum of one-body currents, $J^{+}=\sum_{B, M} J_{B}^{+}+J_{M}^{+}$, which involves individual hadrons one at a time. This corresponds to assuming that there are no interactions among the particles in a multiparticle Fock state during the interaction with the photon. Therefore the external probe can scatter either on the bare nucleon, $|N\rangle$, or one of the constituents of the higher Fock states, $|B M\rangle$. As a result, the matrix elements of the current can be written as the sum of the following two contributions

$$
\left\langle\tilde{p}_{N}^{\prime}, \lambda_{N}^{\prime}, \tilde{N}\left|J_{V}^{+}\right| \tilde{p}_{N}, \lambda_{N}, \tilde{N}\right\rangle=Z I_{\lambda_{N}^{\prime}, \lambda_{N}}^{N}+\delta I_{\lambda_{N}^{\prime}, \lambda_{N}}
$$


In Eq. (4) $I^{N}$ is the contribution from the bare nucleon, $Z$ is the probability of finding a bare nucleon in the physical nucleon, and $\delta I$ is the contribution from the $B M$ Fock components of the physical nucleon. This last term can further be split into two contributions, with the active particle being the baryon $\left(\delta I^{\left(B^{\prime} B\right) M}\right)$ or the meson $\left(\delta I^{\left(M^{\prime} M\right) B}\right)$, i.e.

$$
\delta I_{\lambda_{N}^{\prime}, \lambda_{N}}=\sum_{B, B^{\prime}, M} \delta I_{\lambda_{N}^{\prime}, \lambda_{N}}^{\left(B^{\prime} B\right) M}+\sum_{M^{\prime}, M, B} \delta I_{\lambda_{N}^{\prime}, \lambda_{N}}^{\left(M^{\prime} M\right) B} .
$$

The two terms in Eq. (5) can explicitly be obtained by folding the current matrix elements of the baryon and meson constituents with the probability amplitudes describing the distributions of these constituents in the dressed initial and final nucleon. Furthermore, by using the kinematical nature of the light-front boost, the baryon and meson current matrix elements can be factor out of the internal momentum integration, and the final results read

$$
\begin{aligned}
& \delta I_{\lambda_{N}^{\prime}, \lambda_{N}}^{\left(B^{\prime} B\right) M}\left(Q^{2}\right)=\sum_{\lambda, \lambda^{\prime}, \lambda^{\prime \prime}} I_{\lambda^{\prime} \lambda}^{B^{\prime} B}\left(Q^{2}\right) \int \mathrm{d} y_{B} \int \frac{\mathrm{d}^{2} \mathbf{p}_{B \perp}}{2(2 \pi)^{3}} \\
& \times \phi_{\lambda^{\prime \prime} \lambda}^{\lambda_{N}(N, B M)}\left(y_{B}, \mathbf{k}_{B \perp}\right)\left[\phi_{\lambda^{\prime \prime} \lambda^{\prime}}^{\lambda_{N}^{\prime}\left(N, B^{\prime} M\right)}\left(y_{B}, \mathbf{k}_{B^{\prime} \perp}^{\prime}\right)\right]^{*} \\
& \delta I_{\lambda_{N}^{\prime}, \lambda_{N}}^{\left(M^{\prime} M\right) B}\left(Q^{2}\right)=\sum_{\lambda, \lambda^{\prime}, \lambda^{\prime \prime}} I_{\lambda^{\prime} \lambda}^{M^{\prime} M}\left(Q^{2}\right) \\
& \times \int \mathrm{d} y_{M} \int \frac{\mathrm{d}^{2} \mathbf{p}_{M \perp}}{2(2 \pi)^{3}} \phi_{\lambda^{\prime \prime} \lambda}^{\lambda_{N}(N, B M)}\left(1-y_{M},-\mathbf{k}_{M \perp}\right) \\
& \times\left[\phi_{\lambda^{\prime \prime} \lambda^{\prime}}^{\lambda_{N}^{\prime}\left(N, B M^{\prime}\right)}\left(1-y_{M},-\mathbf{k}_{M^{\prime} \perp}^{\prime}\right)\right]^{*}
\end{aligned}
$$

where $\mathbf{k}_{B(M) \perp}=\mathbf{p}_{B(M) \perp}-\left(1-y_{B(M)}\right) \mathbf{q}_{\perp} / 2$ and $\mathbf{k}_{B(M) \perp}^{\prime}=$ $\mathbf{p}_{B(M) \perp}+\left(1-y_{B(M)}\right) \mathbf{q}_{\perp} / 2$. In Eqs. (6) and (7), the function $\phi_{\lambda^{\prime \prime} \lambda^{\prime}}^{\lambda_{N}(N B, M)}\left(y, \mathbf{k}_{\perp}\right)$ is the probability amplitude to find a physical nucleon with helicity $\lambda_{N}$ in a state consisting of a virtual baryon $B$ and a virtual meson $M$, with the baryon having helicity $\lambda^{\prime \prime}$, longitudinal momentum fraction $y$ and transverse momentum $\mathbf{k}_{\perp}$, and the meson having helicity $\lambda^{\prime}$, longitudinal momentum fraction $1-y$ and transverse momentum $-\mathbf{k}_{\perp}$. In Eq. (5) the sum over all the possible $B M$ configurations leads to contributions from both the diagonal current matrix elements with the same hadrons in the initial and final state $\left(B^{\prime}=B\right.$ and $M^{\prime}=M$ in Eq. (6) and (7), respectively), and the current matrix elements involving the transition between different hadron states (i.e. the terms with $B^{\prime} \neq B$ and $M^{\prime} \neq M$ in Eq. (6) and (7), respectively). Finally, the current matrix elements between the bare-hadron states are calculated as overlap integrals of the hadron LCWFs, corresponding to the minimal Fock-state composition of valence quarks.

\section{Results and discussion}

The instant-form wave function of the bare-hadron states is constructed as a product of a momentum wave function, which is spherically symmetric and invariant under permutations, and a spin-isospin wave function, which is uniquely determined by $\mathrm{SU}(6)$ symmetry requirements. The transformation to the light-cone form is performed via Melosh rotations which break the SU(6) symmetry and introduce components with orbital angular momentum different from zero, as explained in Ref. [7,8]. The functional form of the momentum wave functions is given in Ref. [7], and it depends on three free parameters fitted to the proton $\left(\mu^{p}\right)$ and neutron $\left(\mu^{n}\right)$ anomalous magnetic moments, the axial coupling constant of the proton $g_{A}^{p}=G_{A}^{p}(0)$, and the nucleon electromagnetic form factors at $Q^{2}=0.15$ and $0.45 \mathrm{GeV}^{2}$. The fitting procedure is performed by allowing a $5 \%$ uncertainty. For the anomalous magnetic moments we find $\mu^{p}=2.87$ and $\mu^{n}=-1.80$, with a contribution from the meson cloud equal to $12 \%$ and $15 \%$, respectively, while the result for the axial coupling constant is $g_{A}^{p}=1.20$ with a smaller contribution from the meson cloud of about $6 \%$. These results are pretty close to the experimental values $\mu^{p}=2.79, \mu^{n}=-1.913$ and $g_{A}^{p}=1.267$, and especially for $\mu^{n}$ represent a significant improvement with respect to constituent quark model calculations with only the contribution from the three valence quarks [11,12,13.

In Fig. 1 the results for the magnetic $G_{M}=F_{1}-$ $Q^{2} /\left(4 M_{N}^{2}\right) F_{2}$ and electric $G_{E}=F_{1}+F_{2}$ form factors are shown in comparison with the experimental data. A rather good fit is obtained in the proton case in the whole range of available data, while in the neutron case the fit is less satisfactory. In any case, the contribution from the meson cloud is smooth and only significant for $Q^{2}<0.5 \mathrm{GeV}^{2}$
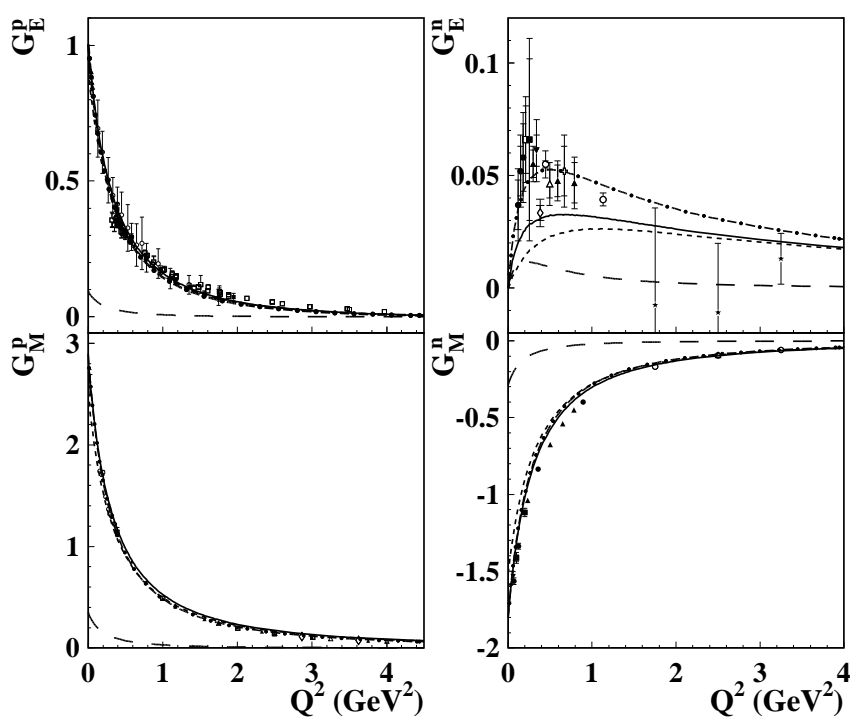

Fig. 1. The four nucleon electromagnetic form factors compared with the world data. considered in the analysis of Ref. [9] and the recent JLab data [10] using $G_{E}^{p}=\left(\mu^{p} G_{E}^{p} / G_{M}^{p}\right) /(1+$ $\left.Q^{2} / 0.71 \mathrm{GeV}^{2}\right)^{2}$ (open squares). Dotted curve for the contribution of the meson cloud; dashed curve for the valence-quark contribution with $\mathrm{SU}(6)$ instant-form nucleon wave function; solid curve for the sum of the two contributions; dashed-dotted curve for the total result with $1 \%$ mixed-symmetry $S^{\prime}$-state in the bare nucleon wave function. 


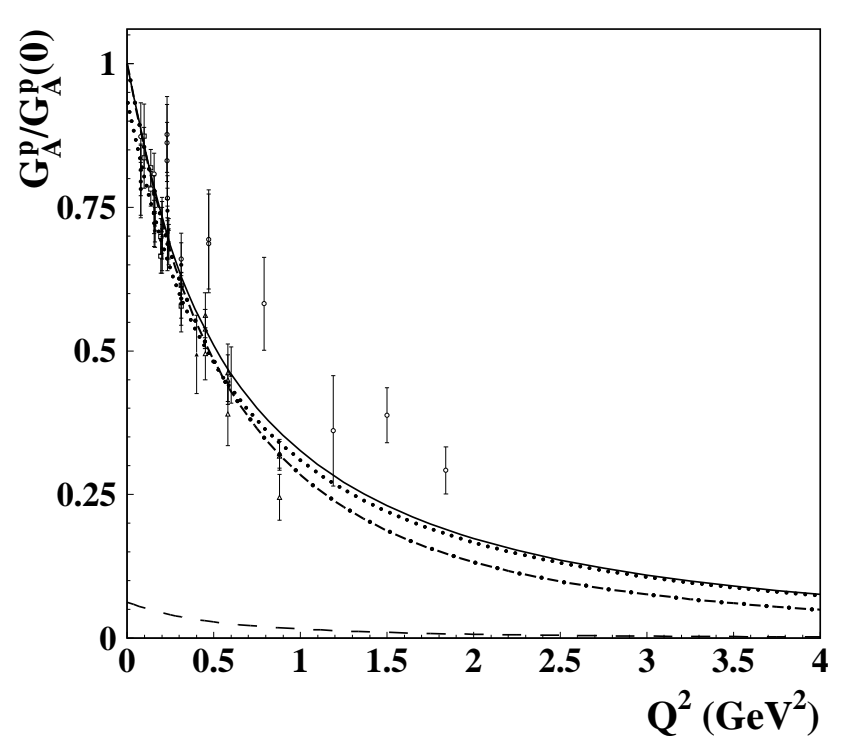

Fig. 2. The axial form factor of the proton. Dotted (dashed) curve for the contribution of the meson cloud (valence quarks). Solid curve for the total result. Dot-dashed curve for the phenomenological dipole form. Data points are the world data considered in Ref. [14].

with a maximum at $Q^{2}=0$. In order to improve the result for the neutron electric form factor we also included a $1 \%$ admixture of mixed-symmetry $S^{\prime}$-wave components, along the lines of Ref. [11. The contribution from such mixed-symmetric component is hardly visible in the case of the proton form factors and the neutron magnetic form factor, while it appreciably improves the agreement with the experimental data in the case of $G_{E}^{n}$.

The predicted axial form factor of the proton is shown in Fig. 2. The meson-cloud contribution is only significant at low values of $Q^{2}$, and the observed dipole form of the axial form factor, i.e. $G_{A}^{p}\left(Q^{2}\right) / G_{A}^{p}(0)=1 /\left(1+Q^{2} / M_{A}^{2}\right)^{2}$ with $M_{A}=1.069 \mathrm{GeV}$, is well reproduced.

The two-dimensional Fourier transform of $F_{1}$ in the transverse plane perpendicular to the direction of motion of the nucleon allows us to map the charge density $\rho(b)$ of the nucleon as function of the transverse distance $b$ from the nucleon center. As it was first pointed out in Ref. [15, the main advantage of the two-dimensional Fourier transform is that we can have a probabilistic interpretation for $\rho(b)$, at variance with the three dimensional Fourier transform of Sachs form factors. The corresponding nucleon densities are plotted in the upper panels of Fig. 3. While the negative tail of the neutron distribution has the well known interpretation in terms of the pion cloud, the negative charge density near the origin appears to be mysterious. An intuitive understanding of this result has been recently given in Ref. [16], suggesting that u quark in the neutron have a larger $\mathrm{p}$-wave component than $\mathrm{d}$ quarks, being therefore suppressed at the origin as shown in the lower panels of Fig. 3. When considering also the Fourier transform of the axial form factor, one can define densities for longitudinally polarized quark in a polarized nucleon 17,17, finding that the positive helicity up quarks
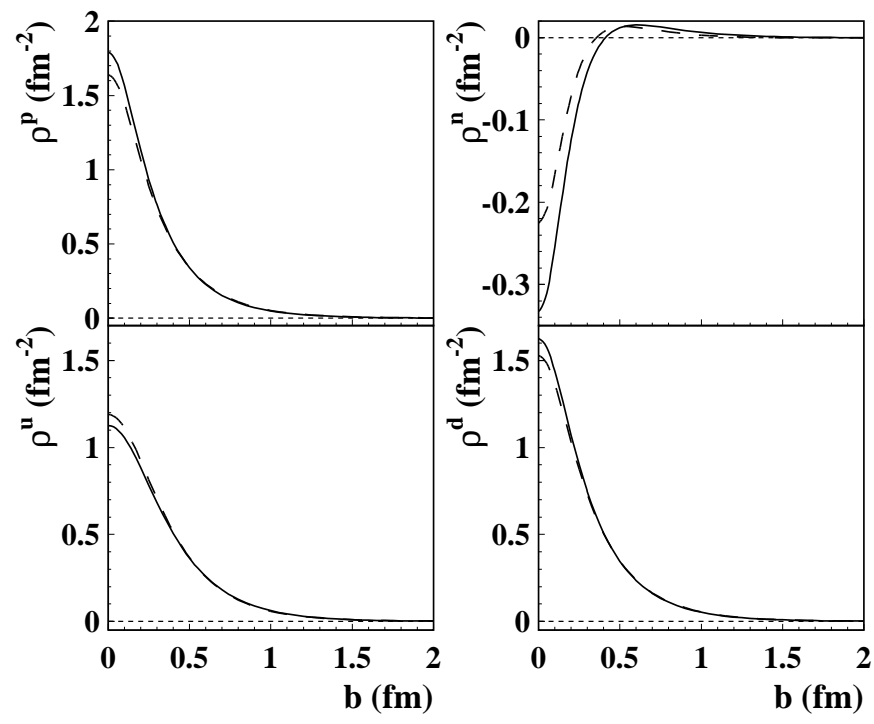

Fig. 3. Upper panels: The proton (left) and neutron (right) charge density as a function of the impact parameter $b$. Lower panels: The up (left) and down (right) transverse charge densities in the neutron. Solid curves for a SU(6)-symmetric instantform wave function, dashed curves with mixed-symmetry components.

in the proton are preferentially aligned with the proton helicity, while the opposite occurs for down quarks.

\section{References}

1. J. Speth, W. A. Thomas, Adv. Nucl. Phys. 24, 83 (1998).

2. Z. Dziembowski, H. Holtmann, A. Szczurek, and J. Speth, Ann. of Phys. 258, 1 (1997).

3. A. Faessler, Th. Gutsche, V.E. Lyubovitskij, and K. Pumsa-ard, Phys. Rev. D 73, 114021 (2006).

4. B. Juliá-Díaz, D.O. Riska, Nucl. Phys. A 780, 175 (2006).

5. D.Y. Chen, Y.B. Dong, M.M. Giannini, and E. Santopinto, Nucl. Phys. A 782, 62c (2007).

6. B. Pasquini, S. Boffi, Phys. Rev. D 73, 094001 (2006); Nucl. Phys. A 782, 86c (2007).

7. B. Pasquini, S. Boffi, Phys. Rev. D 76, 074011 (2007).

8. S. Boffi, B. Pasquini, and M. Traini, Nucl. Phys. B 649, 243 (2003).

9. J. Friedrich, Th. Walcher, Eur. Phys. J. A 17, 607 (2003).

10. V. Punjabi et al. (Jefferson Lab Hall A Collaboration), Phys. Rev. C 71, 055202 (2005); (E) Phys. Rev. C 71, 069902 (2005).

11. B. Juliá-Díaz, D.O. Riska, and F. Coester, Phys. Rev. 69, 035212 (2004); (E) Phys. Rev. C 75, 069902 (2005).

12. R.F. Wagenbrunn, S. Boffi, W. Klink, W. Plessas, and M. Radici, Phys. Lett. B 511, 33 (2001).

13. F. Cardarelli, E. Pace, G. Salmè, and S. Simula, Phys. Lett. B 357, 267 (1995).

14. V. Bernard, L. Elouadrhiri, and Ulf-G. Meißner, J. Phys. G: Nucl. Part. Phys. 28, R1 (2002).

15. G.A. Miller, Phys. Rev. Lett. 99, 112001 (2007).

16. M. Burkardt, arXiv:0709.2966 [hep-ph].

17. B. Pasquini, S. Boffi, Phys. Lett. B 653, 23 (2007). 